\title{
Penetrating cardiac stab wounds: A case report with management algorithm and review of the literature
}

\author{
Antonino M. Grande, ${ }^{1}$ Filippo Antonacci, ${ }^{2}$ Paolo Aseni ${ }^{3}$ \\ ${ }^{1}$ Cardiac Surgery, IRCCS Fondazione Policlinico San Matteo, Pavia; ${ }^{2}$ Thoracic Surgery, IRCCS \\ Fondazione Policlinico San Matteo, Pavia; ${ }^{3}$ Emergency Department and Urgency Medicine Ward, ASST \\ Grande Ospedale Metropolitano, Niguarda, Milan, Italy
}

\begin{abstract}
A 57-year-old man attempted a suicide self-inflicting multiple scissors stab wounds in the chest. At the scene, Focused Assessment with Sonography in Trauma (FAST) showed an important left pleural effusion and pericardial fluid. Computed Tomography Angiography confirmed the pericardial effusion. The patient underwent immediate surgery. Three epicardial wounds of the anterior surface of the right ventricle were identified, one of which was actively bleeding. The lesion was sutured, the patient recovered uneventfully, and on the sixth postoperative day was transferred to a psychiatric unit. At 6-month follow-up, he is doing well and has returned to work. We discuss the importance of FAST for an early diagnosis of chest penetrating trauma leading to a rapid life-saving cardiac procedure and propose a clinical-based protocol for the management of patients with suspected penetrating cardiac injury which we have applied in our service for last six years. Midline sternotomy should be considered the incision of choice in patients with penetrating trauma in the cardiac box with evidence of injury to the heart and great vessels. Emergency Department Thoracotomy can be a possible option for those patients with impending cardiac arrest despite adequate resuscitation.
\end{abstract}

Correspondence: Antonino M. Grande, Cardiac Surgery, IRCCS Fondazione Policlinico San Matteo P.le C. Golgi, 2, 27100 Pavia, Italy. Tel.: +39.0382.503515.

E-mail: amgrande@libero.it

Key words: Penetrating cardiac trauma; Thoracic trauma; Extended focused abdominal sonography for trauma.

Contributions: the authors contributed equally.

Conflict of interest: the authors declare no potential conflict of interest.

Funding: none.

Received for publication: 26 February 2018.

Revision received: 15 June 2018.

Accepted for publication: 15 June 2018.

This work is licensed under a Creative Commons Attribution 4.0 License (by-nc 4.0).

(C) Copyright A.M. Grande, et al., 2018

Licensee PAGEPress, Italy

Emergency Care Journal 2018; 14:7384

doi:10.4081/ecj.2018.7384

\section{Introduction}

Penetrating cardiac injuries (PCIs) are exceedingly devastating lesions that represent a major surgical challenge with a high mortality rate ranging from 40 to $90 \% .{ }^{1-11}$ Cardiac stab wounds are considered a real challenge in the Emergency Department because of their unpredictable clinical course and the need for immediate diagnosis and emergency care. In PCI the right ventricle is involved more often than the left ventricle because it is positioned anteriorly in the chest and occupies the largest part of the anterior surface of the heart. A growing number of innovative diagnostic and therapeutic approaches have been introduced during last decade which have contributed to a significant amelioration in patients' survival. We present a case of a patient with stab wounds located in the left hemithorax who reported a right ventricle injury. We also discuss our Extended Focused Assessment with Sonography in Trauma(EFAST)-based algorithm for early diagnosis and management of penetrating thoracic injuries, which is based on the clinical variables at higher risk for immediate death.

\section{Case Report}

A 57-year-old man attempted suicide by self-inflicting scissors stab wounds in the chest. He was found unconscious, lying on the floor and in a pool of blood. On the ambulance, FAST showed an important left pleural effusion and pericardial fluid; arterial systolic blood pressure was $100 \mathrm{mmHg}$ and cardiac rate $110 / \mathrm{min}$. Arriving in the Emergency Department (ED), he presented three scissors stab wounds medially to the left nipple (Figure 1). He was diaphoretic, tachypneic, and tachycardic (HR 105/min); his systolic blood pressure was $80-90 \mathrm{mmHg}$, no jugular vein distension was observed. Volume replacement with $1500 \mathrm{~mL}$ of crystalloids resulted in some improvement of systolic blood pressure. EFAST evaluation, in the hands of the emergency physician, confirmed an important left pleural effusion and a mild pericardial effusion. In consideration of the relative hemodynamic stability after volume replacement, we decided to proceed to a Computed Tomography Angiography (CTA) that, in addition to the known pleural and pericardial effusion, showed the presence of air in the anterior mediastinum and in the left pleural cavity (Figure 2). After CTA, systolic blood pressure dropped again to $60-70 \mathrm{~mm} \mathrm{Hg}$, and the patient was immediately operated upon. A left anterolateral thoracotomy in the fifth intercostal space was performed, and $800 \mathrm{~mL}$ of blood were evacuated from the left chest; the left lung was deflated to open the pericardium causing a further drop in blood pressure. The pericardium was then opened, and a blood clot (150 $\mathrm{mL}$ ) was removed. Three epicardial wounds were found on the anterior surface of the right ventricle, one of which, $1 \mathrm{~cm}$ beside 
the left anterior coronary artery was actively bleeding. The bleeding lesion was sutured using 4.0 polypropylene reinforced with Teflon strip (Figure 3). Three pulmonary parenchymal lesions (Figure 4) were directly repaired on the anterior aspect of the superior lobe of the left lung using polypropylene 4.0.

The patient recovered uneventfully, and on the sixth postoperative day, was transferred to a psychiatric unit. At 14-month follow-up, he is doing well and has returned to work.

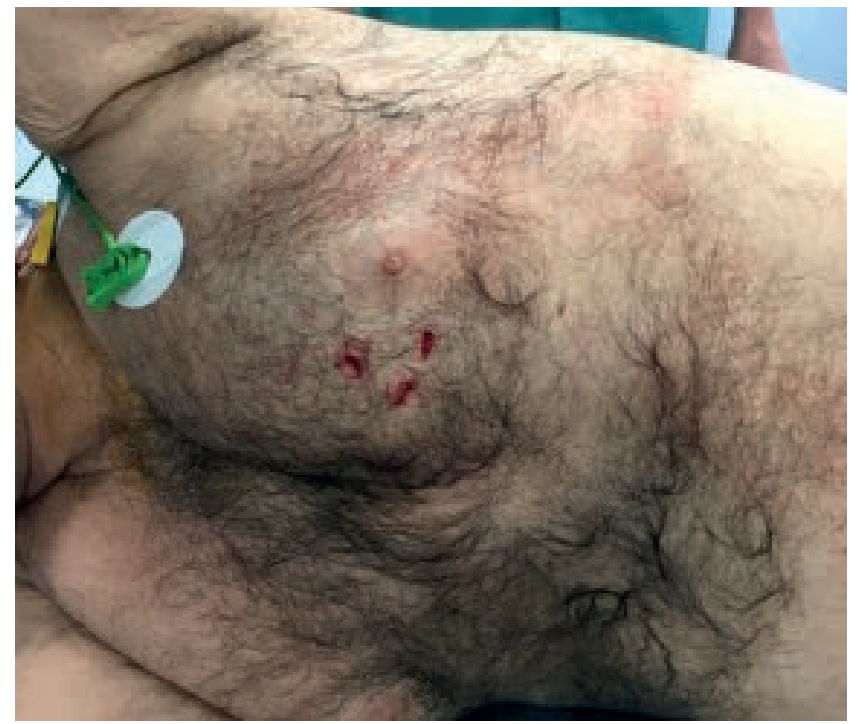

Figure 1. Self-inflicted scissors stab wound in a 57-year-old man who attempted suicide: three scissor stab wounds are visible medially the left nipple.

\section{Discussion}

Major and life-threatening chest injuries can be memorized as the Deadly Dozen. The first six are lethal and require immediate evaluation and treatment during primary survey: airway obstruction, tension pneumothorax, cardiac tamponade, open pneumothorax, massive hemothorax, and flail chest. The second six are hid-

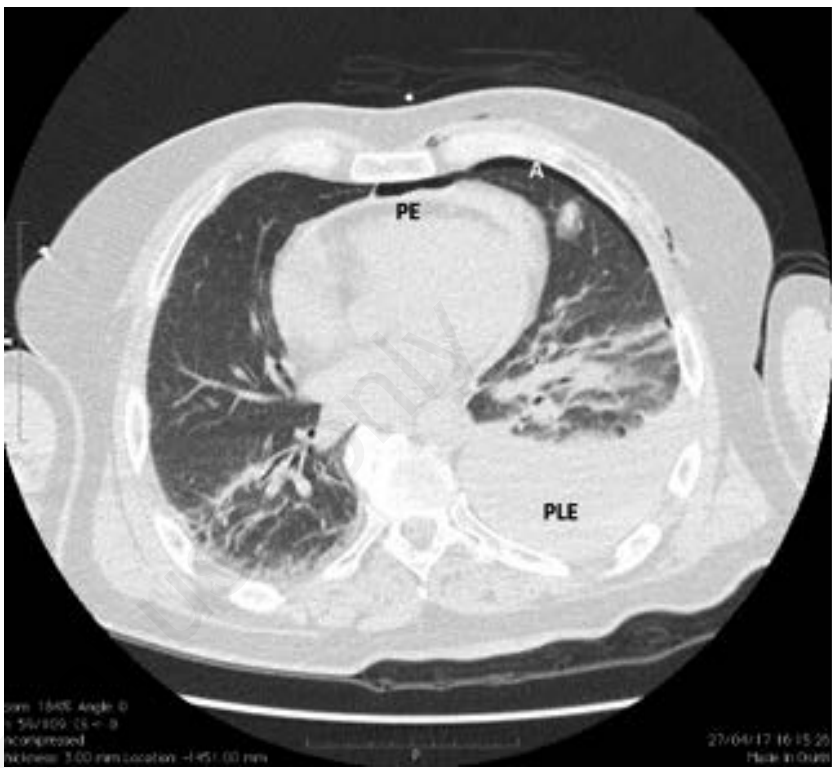

Figure 2. Chest CT scan showing pericardial effusion (PE), a huge left pleural effusion (PLE) and air (A) in the anterior mediastinum and anteriorly in the left pleural cavity.

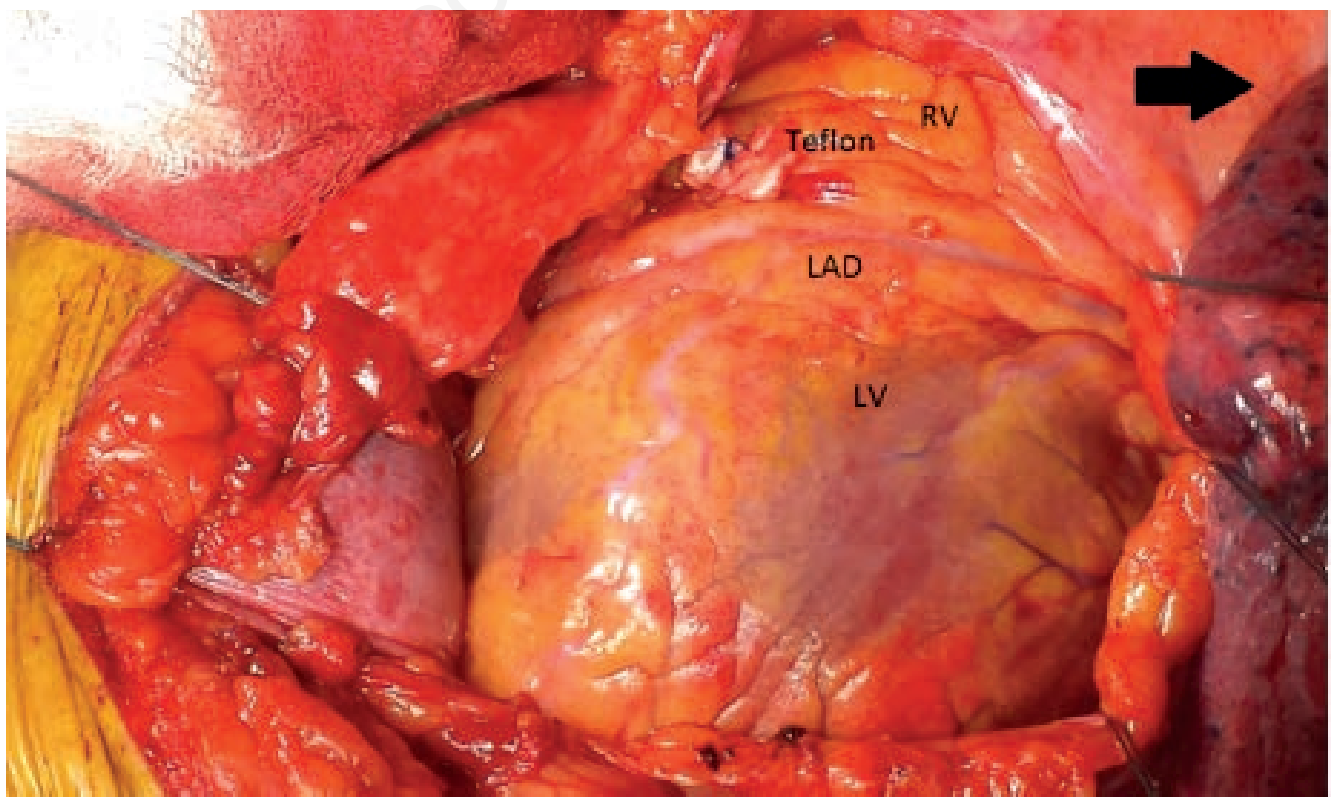

Figure 3. Left anterolateral thoracotomy, in fourth intercostal space, suture of one right ventricle lesion with polypropylene 4.0 reinforced by Teflon strip. RV, right ventricle; LAD, left anterior descending coronary artery; LV, left ventricle; the black arrow indicates patient's head. 
den: thoracic aortic disruption, tracheobronchial disruption, myocardial contusion, traumatic diaphragmatic tear, esophageal disruption, and pulmonary contusion. The hidden six are potentially life-threatening injuries and are usually diagnosed at a later survey. ${ }^{12,13}$

Thoracic injuries should be organized into 4 groups according to the topographical site of the wounds or trajectory of the knife or missile: ${ }^{12}$ i) base of the neck into mediastinum or pleural cavity; ii) one pleural cavity into the mediastinum or spinal cord; iii) parasternal wound; iv) two lateral chest wounds involving both pleural cavities and mediastinum (usually caused by gunshot). It is very important not to assume a straight line of missile's trajectory because surface injuries often do not correspond to missile trajec-

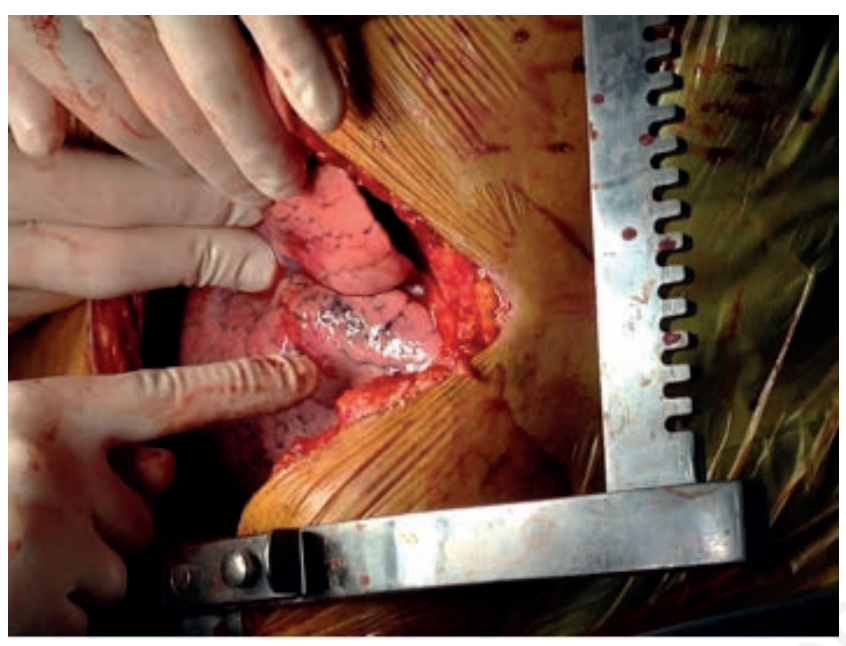

Figure 4. Left superior lobe injuries. tory. This consideration was also true in our case where left hemithorax injuries determined a right ventricle damage.

PCIs are present in about $6.4 \%$ of all penetrating chest injuries,${ }^{14}$ and are considered a real challenge in the ED because of their unpredictable clinical course and the need for immediate diagnosis and emergency care. For this reason, an injury to the heart should be immediately suspected in any patient with penetrating trauma to the chest. Considering that the diaphragm is a

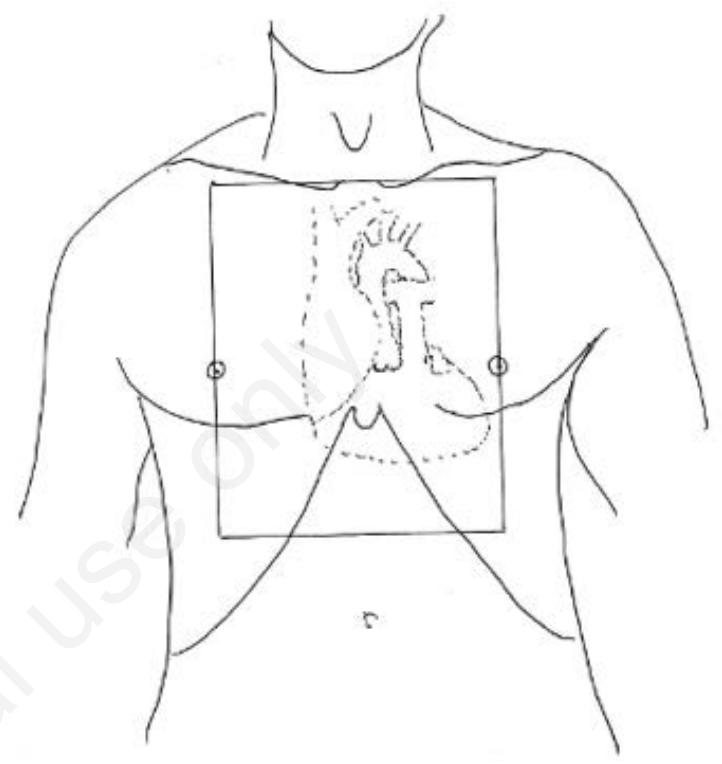

Figure 5. The cardiac box: the area is delimited superiorly by the sternal notch, laterally by the nipples and inferiorly by a transverse line halfway between the xiphoid process and the umbilicus.

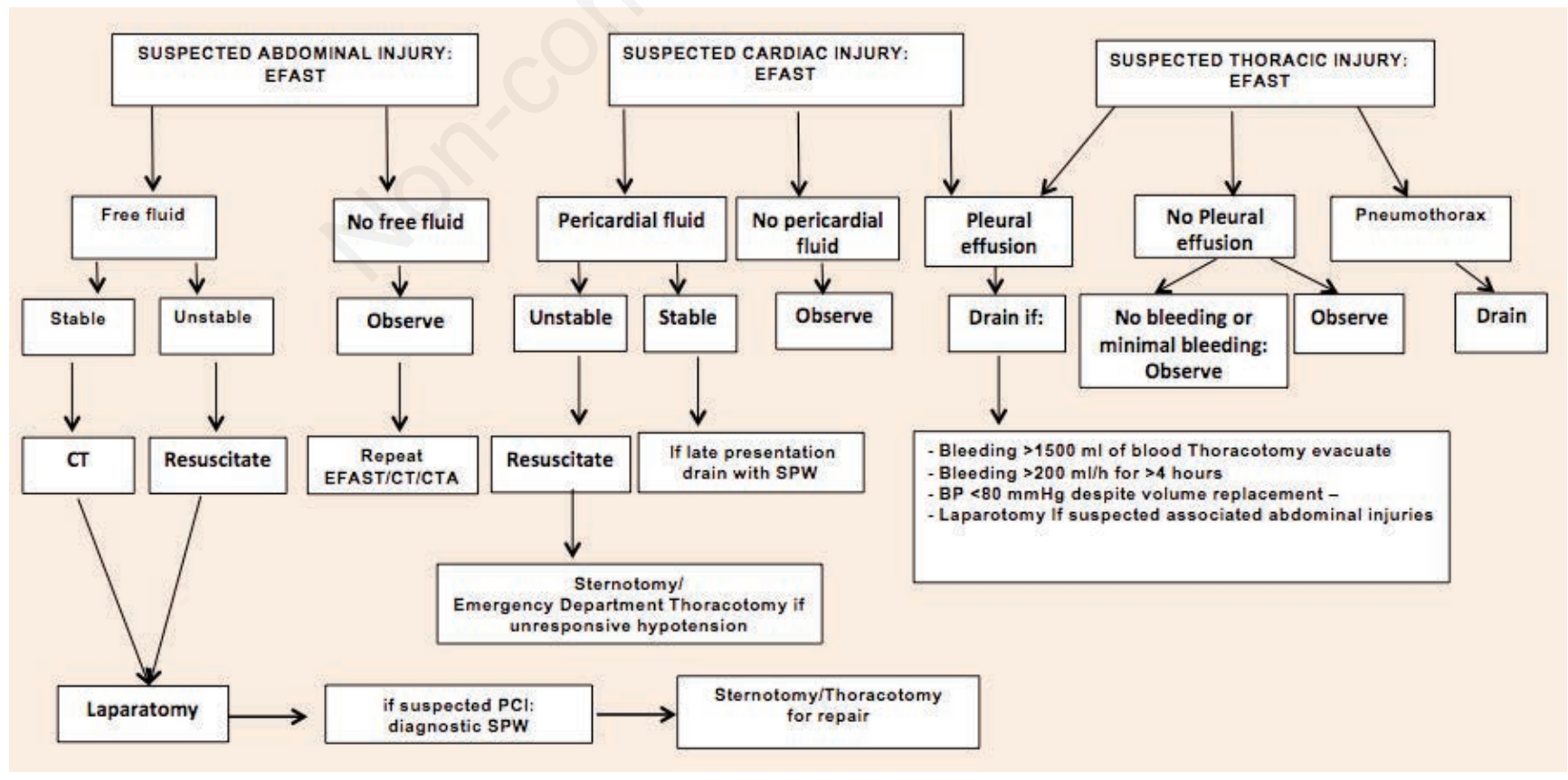

Figure 6. Clinical-based EFAST algorithm in penetrating trauma. SPW, Subxyphoid Pericardial Window; CT, Computerized Tomography; CTA, Computerized Tomography Angiography; PCI, Penetrating Cardiac Injury; EFAST, Extended Focused Assessment with Sonography in Trauma. 
moving target, an injury that at first glance may look abdominal, can actually be intrathoracic and vice versa. Physical examination in a patient presenting external chest wounds may help to find markers for possible internal injuries. High suspicion for cardiac injury is necessary when a wound occurs within the so-called cardiac box (Figure 5), a region delimited superiorly by the sternal notch, laterally by the nipples and inferiorly by the trans-pyloric line (a transverse line halfway between the xiphoid process and the umbilicus). The clinical presentation of PCI may range from hemodynamic compensatory stability to instability and cardiopulmonary arrest and can be related to several factors, including wounding mechanism, length of time elapsed before arrival in the trauma center, and extent of the injury. The muscular nature of the left ventricle, and to a lesser extent of the right ventricle, may seal myocardial penetrating injuries and prevent an exsanguinating haemorrhage, allowing some patients to arrive haemodynamically stable to the Emergency center, sometimes with a deceptive clinical presentation.

Most patients with a PCI are unstable, and many arrive in the emergency department receiving cardiopulmonary resuscitation. If the PCI is extensive, the injury will invariably lead to exsanguinating hemorrhage into the left hemithoracic cavity and the patient will develop a cardiopulmonary arrest. About $80 \%$ to $90 \%$ of patients with cardiac stab wounds present with signs of tamponade. ${ }^{15}$ Nevertheless, Beck's triad (muffled heart tones, jugular venous distention, and hypotension), as well as Kussmaul's sign (jugular venous distention upon inspiration) represent the exception rather than the rule being present in only approximately $10 \%$ of patients with PCI. While pericardial tamponade may have a protective action by preventing a massive haemothorax, it may also be a harbinger of shock because it decreases ventricular filling, stroke volume and cardiac output. Mild to moderate amounts of cardiac tamponade can be temporarily overcome by rapid fluid administration that increases preload and cardiac output, while waiting for definitive surgical treatment. Needle pericardiocentesis is of limited benefit for the diagnosis and reversal of a tamponade. Penetrating the needle into a cardiac chamber may determine a false-positive result, while a false negative (up to $80 \%$ in some series) may be caused by clotting of the blood in the pericardial cavity. ${ }^{15}$ This maneuver maintains a role only when no surgeon, or operating room is available ${ }^{15}$ because a decompressing procedure can save time to get the patient to surgery or to a trauma center. As is often the case in surgical practice, new techniques and investigations are available, and a growing number of innovative diagnostic and therapeutic approaches have been introduced in recent years, determining a significant improvement in survival.

Recent works ${ }^{16}$ have renewed interest in the use of the subxiphoid pericardial window (SPW) not only to confirm or exclude the presence of a hemopericardium but also as a therapeutic procedure in selected patients.

Clinical echography, in the form of EFAST, has come to represent the preferred initial examination in patients with PCI, because of its simplicity, rapidity, and accuracy. Sensitivity of EFAST for the presence of pericardial fluid is very high with reported values of $97-100 \% .{ }^{17}$ It is possible to perform FAST at the scene of an accident, and the test can be accomplished by both paramedics and physicians after an appropriate training period and a relatively low number of supervised exams. A negative clinical examination after primary survey in patients with PCI does not necessarily exclude a cardiac injury, and EFAST should be repeated since injuries which are not immediately detectable can at times become evident even after 24 hours

In this light, we have reviewed our management of patients with penetrating injuries, elaborating an algorithm that we have used for the last 6 years which incorporates both EFAST and SPW (Figure 6). In our protocol, SPW is reserved to a particular subset of patients with delayed presentation of traumatic pericardial effusion without hemodynamic instability and may have a role in excluding the presence of a cardiac injury in patients requiring laparotomy for abdominal trauma. Our predominantly clinically driven approach considers site of injury, hemodynamic status, chest radiograph findings, EFAST, arterial blood gases, prompt review and requires an immediate coordination between experienced emergency physicians and trauma team surgeons.

Midline sternotomy should be considered the incision of choice in the emergency cases with resuscitative purposes when a penetrating trauma in the cardiac box may determine an injury to the heart or great vessels. In our case, the presence of a huge left pleural effusion induced the thoracic surgeon to choose a left anterior surgical approach in the assumption that this could be the most important lesion. This approach determined an optimal left lung exposure; however, when the pericardium was opened, and the right ventricle lesions were identified, cardiac exposure was much more difficult to achieve. Indeed a midline sternotomy would have resulted in a much easier surgical procedure.

In pulseless patients presenting to the emergency department with signs of life after penetrating thoracic injury, some authors strongly recommend Emergency Department Thoracotomy (EDT). Recommendation for EDT is based on moderate quality of evidence for those patients with cardiac arrest but with previously witnessed cardiac activity or with unresponsive hypotension $(\mathrm{BP}<70 \mathrm{mmHg})$ despite adequate resuscitation. The procedure requires a supine left antero-lateral thoracotomy in the emergency room. The incision, which can be prolonged across the sternum in a clamshell incision, allows fast release of cardiac tamponade, control of cardiovascular haemorrhage, internal cardiac massage and aortic cross-clamping of the descending thoracic aorta. ${ }^{18}$

\section{Conclusions}

PCI are highly lethal, and a high index of suspicion, coupled with early operative intervention, remains the key to the survival of these patients. EFAST has become the most common initial screening modality in the majority of trauma centers worldwide, and it is included in the Advanced Trauma Life Support program for evaluation of the hypotensive trauma patient. ${ }^{19-21}$ Midline sternotomy should be considered the incision of choice in the emergency setting in patients with penetrating trauma in the cardiac box. EDT in the emergency room can be a lifesaving procedure in selected patients with hemodynamic collapse.

\section{References}

1. Campbell NC, Thomson SR, Muckart DJ. Review of 1198 cases of penetrating cardiac trauma. Br J Surg 1997;84:173740.

2. Kish G, Kozloff L, Joseph WL, Adkins PC. Indications for early thoracotomy in the management of chest trauma. Ann Thorac Surg 1976;22:23-8.

3. Demetriades D, van der Veen BW. Penetrating injuries of the heart: experience over two years in South Africa. J Trauma 1983;23:1034-41.

4. Asensio JA, Soto SN, Forno W, et al. Penetrating cardiac 
injuries: a complex challenge. Injury 2001;32:533-43.

5. Asensio JA, Berne JD, Demetriades D, et al. One hundred five penetrating cardiac injuries: a 2-year prospective evaluation. J Trauma 1998;44:1073-82.

6. Thourani VH, Feliciano DV, Cooper WA, et al. Penetrating cardiac trauma at an urban trauma center: a 22-year perspective. Am Surg 1999;65:811-6.

7. Degiannis E, Loogna P, Doll D. Penetrating cardiac injuries: recent experience in South Africa. World J Surg 2006;30:125864.

8. Gao JM, Gao YH, Wei GB, et al. Penetrating cardiac wounds: principles for surgical management. World J Surg 2004;28:1025-9.

9. Asensio JA, Stewart BM, Murray J, et al. Penetrating cardiac injuries. Surg Clin North Am 1996;76:685-724.

10. Asensio JA, Murray J, Demetriades D, et al. Penetrating cardiac injuries: a prospective study of variables predicting outcomes. J Am Coll Surg 1998;186:24-34.

11. Demetriades D, Rabinowitz B, Markides N. Indications for thoracotomy in stab injuries of the chest: a prospective study of 543 patients. Br J Surg 1986;73:888-90.

12. Burack JH, Kandil E, Sawas A, et al. Triage and outcome of patients with mediastinal penetrating trauma. Ann Thorac Surg 2007;83:377-82.

13. Ivatury R, Rohman M. Penetraing cardiac trauma. In: Turney SZ, Rodriguez A, Cowley RA, eds. Management of cardiothoracic trauma. Baltimore (MD): Williams \& Wilkins; 1990. pp
311-327.

14. Mandal AK, Sanusi M. Penetrating chest wounds: 24 years experience. World J Surg 2001;25:1145-9.

15. When-Yen C, Jane-Yi H, Yee-Phoung C, et al. The successful management of a penetrating cardiac Injury in a regional hospital: A case report. J Emerg Crit Care Med 2008;19:160-5.

16. Nicol AJ, Navsaria PH, Hommes M, et al. Sternotomy or drainage for a hemopericardium after penetrating trauma: a randomized controlled trial. Ann Surg 2014;259:438-42.

17. Adams A, Fotiadis N, Chin JY, et al. A pictorial review of traumatic pericardial injuries. Insight Imaging 2012;3:307-11.

18. Seamon MJ, Haut ER, Van Arendonk K, et al. An evidencebased approach to patient selection for emergency department thoracotomy: A practice management guideline from the Eastern Association for the Surgery of Trauma. J Trauma Acute Care Surg 2015;79:159-73.

19. Boulanger BR, Kearney PA, Brenneman FD, et al. Utilization of FAST (Focused Assessment with Sonography for Trauma) in 1999: results of a survey of North American trauma centers. Am Surg 2000;66:1049-55.

20. Kool DR, Blickman JG. Advanced Trauma Life Support. ABCDE from a radiological point of view. Emerg Radiol 2007; $14: 135-41$.

21. Richards JR, McGahan JP. Focused Assessment with Sonography in Trauma (FAST) in 2017: What Radiologists Can Learn. Radiology 2017;283:30-48. 